\title{
POSITIVE SOLUTIONS FOR A SECOND ORDER EXTENDED FISHER-KOLMOGOROV'S EQUATION
}

Diko Souroujon $^{1}$, Stepan Tersian ${ }^{2} \S$

${ }^{1}$ University of Economics, Varna

Department of Mathematics

Kniaz Boris I Bvd. 77, Varna - 9002, BULGARIA

${ }^{2}$ Institute of Mathematics and Informatics

Bulgarian Academy of Sciences

Acad. G. Bonchev Str., Block 8, Sofia - 1113, BULGARIA

Abstract: We consider the existence of positive solutions of extended FisherKolmogorov second order differential equation. Using a variational method and an approach of Verzini, we obtain the positive bounded solutions of this ODE.

AMS Subject Classification: 34C37, 34G20, 49J27, 49J05

Key Words: positive solutions; Fisher-Kolmogorov equation; Sobolev space; variational method

\section{Introduction}

In the present paper we study the existence of positive solutions of a secondorder ordinary differential equation (ODE)

$$
u^{\prime \prime}+c u^{\prime}+f(t, u)=0, \quad t \in(a,+\infty),
$$

coupled with the boundary conditions

$$
u(a)=u(+\infty)=0,
$$

where $c>0$ is a constant, $a \in \mathbb{R}$. We suppose that $f(t, s): \mathbb{R}^{2} \rightarrow \mathbb{R}$ and

Received: September 20, 2020

(C) 2020 Academic Publications

${ }^{\S}$ Correspondence author 
$f_{s}(t, s)=\frac{\partial f}{\partial s}(t, s), f_{t}(t, s)=\frac{\partial f}{\partial t}(t, s)$ are continuous functions which satisfy the following conditions:

$$
\begin{gathered}
c_{1}|s|^{1+q} \leq|f(t, s)| \leq c_{2}|s|^{1+q}, s f(t, s) \geq 0, \quad \forall(t, s) \in \mathbb{R}^{2}, \\
f_{s}(t, s) s^{2}-A f(t, s) \geq 0, \quad \forall(t, s) \in \mathbb{R}^{2}, \\
f(t, s) s-(2+\alpha) F(t, s) \geq 0, \quad \forall(t, s) \in \mathbb{R}^{2}, \\
c_{3}|s|^{q+2} \geq f_{t}(t, s) s \geq 0, \quad \forall(t, s) \in \mathbb{R}^{2},
\end{gathered}
$$

where $A>1, \alpha>0, c_{j}>0, j=1,2,3$, and $F(t, s):=\int_{0}^{s} f(t, \tau) d \tau$.

An example of a function $f$, which satisfies these conditions is $f(t, s)=$ $C|s|^{q} . s$ with $A \in(1, q+1], \alpha=q, c_{1}=c_{2}=c_{3}=C>0$.

We will look for positive solutions $u$ of (1.1) such that $u \in H_{0}^{1}(a,+\infty) \cap H_{c, a}$, where $H_{0}^{1}(a,+\infty)$ is the usual Sobolev space and

$$
H_{c, a}:=\left\{u \in H_{l o c}^{1}(a,+\infty): \int_{a}^{+\infty} e^{c t} u^{\prime}(t)^{2} d t<+\infty, \quad u(+\infty)=0\right\}
$$

with norm

$$
\|u\|_{c, a}=\int_{a}^{+\infty} e^{c t}\left(u^{\prime}(t)^{2}+u(t)^{2}\right) d t
$$

and $H_{c, a}^{0}:=H_{c, a} \cap H_{0}^{1}(a,+\infty)=\left\{u \in H_{c, a}: u(a)=0\right\}$.

Equation (1) is obtained by the Fisher-Kolmogorov's equation $u_{t}=u_{x x}+$ $f(t, u)$, looking for the traveling waves $u(x, t)=U(x-c t)$ with speed $c$. There is a vast studies on heteroclinic solutions of eq. (1). We refer the reader to Kolmogorov, Petrovsky and Piskunov [3], Aronson and Weinberger [2], Arias and al. [1], Nehari [7], Versini [9], Szulkin [8], Li and Wang [4] and references therain. Solutions of (1) with initial data with compact support are studied in [2]. Fast solutions of Eq. (1) are studied in the paper of Arias and al. [1] via variational methods. Heteroclinic solutions for non-autonomous second order differential equations are studied in $[5,10]$. Verzini also studied equation of type (1), when $c=0$, and she proves the existence of many oscillating solutions belonging to $L^{\infty}(\mathbb{R})$. She used the variational method and the approach of Nehari [7]. In the present paper we will prove the existence of positive solution of Eq. (1), belonging to $H_{0}^{1}(a,+\infty)$, using the methods of [9].

Note that by (H1) it follows

$$
\frac{c_{1}}{q+2}|s|^{q+2} \leq F(t, s) \leq \frac{c_{2}}{q+2}|s|^{q+2}, \quad \forall(t, s) \in \mathbb{R}^{2},
$$

for some positive constants $c_{1}$ and $c_{2}$. 
We introduce the energy functional associated with the problem (1), (2), further referred as $(\mathrm{P})$ :

$$
\begin{gathered}
J_{[a, b]}(u):=\int_{a}^{b} e^{c t}\left(\frac{u^{\prime}(t)^{2}}{2}-F(t, u(t))\right) d t, \\
J(u)=J_{[a,+\infty)}(u):=\int_{a}^{+\infty} e^{c t}\left(\frac{u^{\prime}(t)^{2}}{2}-F(t, u(t))\right) d t,
\end{gathered}
$$

where $a \in \mathbb{R}$ is a fixed real number and $b \in(a,+\infty)$. Let

$$
\mu(u)=\mu_{[a,+\infty)}(u):=\sup _{\lambda>0} J_{[a,+\infty)}(\lambda u)=\sup _{\lambda>0} J(\lambda u) .
$$

We will prove that for each nonzero function $u, \mu(u)=\sup _{\lambda>0} J(\lambda u) \geq C>0$. There exists unique number $\lambda=\lambda(u)>0$, for which $\sup _{\lambda>0} J(\lambda u)$ is attained, i.e. $\mu(u)=\sup _{\lambda>0} J(\lambda u)=J(\lambda(u) u)$.

We introduce the following set, analogous to the Nehari manifold:

$$
\begin{gathered}
N(a,+\infty):=\left\{u \in H_{c, a}^{0} \backslash(0): \lambda(u)=1\right\} \\
=\left\{u \in H_{c, a}^{0} \backslash(0): \nabla J(u) \cdot u=0\right\},
\end{gathered}
$$

as in Verzini [9]. Since we are looking for the solutions of (1), which are nonnegative on $[a,+\infty)$, we introduce the set

$$
N^{+}(a,+\infty):=\{u \in N(a,+\infty): u \geq 0\} .
$$

Define the function

$$
\varphi^{+}(a,+\infty):=\inf \left\{\sup _{\lambda>0} J(\lambda u): u \in H_{c, a}^{0} \backslash(0), u \geq 0\right\} .
$$

Our main result is:

Theorem 1. Let the conditions (H1)-(H4) hold.

Then $\varphi^{+}(a,+\infty)$ is attained by at least one function $u_{+} \in N^{+}(a,+\infty), u_{+}>$ 0 on $(a,+\infty)$ and $u_{+}(t)$ is a solution of the problem $(P)$ for $t \in(a,+\infty)$.

The paper is organized as follows. In Section 2 we give preliminaries on the function spaces, embedding inequalities and three lemmas for the corresponding functional $J$. In Section 3 we give the proof of Theorem 1 and some comments. 


\section{Preliminaries}

By [1] for each $u \in H_{c, a}$ the following inequality holds:

$$
\int_{a}^{+\infty} e^{c t} u^{\prime}(t)^{2} d t \geq \frac{c}{2} e^{c t_{0}} u\left(t_{0}\right)^{2}+\frac{c^{2}}{4} \int_{a}^{+\infty} e^{c t} u(t)^{2} d t
$$

for any $t_{0} \in[a,+\infty)$. The inequality (6) shows that in the linear space $H_{c, a}$ we can introduce the norm

$$
\|u\|_{H_{c, a}}=\left(\int_{a}^{+\infty} e^{c t} u^{\prime}(t)^{2} d t\right)^{\frac{1}{2}}
$$

corresponding to the scalar product $\langle u, v\rangle_{H_{c, a}}=\int_{a}^{+\infty} e^{c t} u^{\prime}(t) v^{\prime}(t) d t$.

For each function $u \in H_{c, a} \subset L^{\infty}[a,+\infty), \sup _{t \in[a,+\infty)}|u(t)|<+\infty$ and it is attained, i.e.

$$
\sup _{t \in[a,+\infty)}|u(t)|=\max _{t \in[a,+\infty)}|u(t)|=\left|u\left(t_{1}\right)\right|
$$

for some point $t_{1} \in[a,+\infty)$. Further by $C$ we will denote various positive constants not depending on $u$.

We have the following lemma.

Lemma 1. Let $u \in H_{c, a}$. Then the inequality

$$
\begin{gathered}
\int_{a}^{+\infty} e^{c t} u^{\prime}(t)^{2} d t \geq C\left(u\left(t_{1}\right)^{2}+\int_{a}^{+\infty} e^{c t} u(t)^{2} d t+\frac{\int_{a}^{+\infty} e^{c t}|u(t)|^{q+2} d t}{\left|u\left(t_{1}\right)\right|^{q}}\right. \\
\left.+\left(\int_{a}^{+\infty} e^{c t}|u(t)|^{q+2} d t\right)^{\frac{2}{q+2}}\right),
\end{gathered}
$$

holds for a constant $C>0$.

Proof. We have by (6) that

$$
\begin{aligned}
& \int_{a}^{+\infty} e^{c t} u^{\prime}(t)^{2} d t \geq \frac{c}{2} e^{c t_{1}} u\left(t_{1}\right)^{2} \\
& \int_{a}^{+\infty} e^{c t} u^{\prime}(t)^{2} d t \geq \frac{c^{2}}{4} \int_{a}^{+\infty} e^{c t} u(t)^{2} d t
\end{aligned}
$$

By Young's inequality we get

$$
\int_{a}^{+\infty} e^{c t} u^{\prime}(t)^{2} d t \geq C\left(u\left(t_{1}\right)^{2}+\int_{a}^{+\infty} e^{c t} u(t)^{2} d t\right)
$$




$$
\begin{aligned}
& \geq C\left(u\left(t_{1}\right)^{2}+\frac{\int_{a}^{+\infty} e^{c t}|u(t)|^{q+2} d t}{\left|u\left(t_{1}\right)\right|^{q}}\right) \\
& \geq C\left(u\left(t_{1}\right)^{2}\right)^{\frac{q}{q+2}} \frac{\left(\int_{a}^{+\infty} e^{c t}|u(t)|^{q+2} d t\right)^{\frac{2}{q+2}}}{\left(\left|u\left(t_{1}\right)\right|^{q}\right)^{\frac{2}{q+2}}} \\
& =C\left(\int_{a}^{+\infty} e^{c t}|u(t)|^{q+2} d t\right)^{\frac{2}{q+2}}, C>0
\end{aligned}
$$

for each nonzero function $u \in H_{c, a}$. These inequalities imply (7).

Next, we have the following

Lemma 2. Let the function $f(t, s) \in C^{1}\left(\mathbb{R}^{2}\right)$ satisfy the conditions (H1), (H4) and $\mu(u)$ is defined by (5). Then, for every nonzero function $u(t) \in H_{c, a}$, $\mu_{[a,+\infty)}(u) \geq C>0$, where the constant $C$ does not depend on $u$.

Proof. By (4),

$$
\begin{aligned}
J(\lambda u) & =\frac{1}{2} \lambda^{2} \int_{a}^{+\infty} e^{c t} u^{\prime}(t)^{2} d t-\int_{a}^{+\infty} e^{c t} F(t, \lambda u(t)) d t \\
& \geq \frac{1}{2} \lambda^{2} \int_{a}^{+\infty} e^{c t} u^{\prime}(t)^{2} d t-\left(\frac{c_{2}}{q+2} \int_{a}^{+\infty} e^{c t}|u(t)|^{q+2} d t\right) \lambda^{q+2} \\
& =A_{1} \lambda^{2}-B \lambda^{q+2},
\end{aligned}
$$

where $A_{1}=\frac{1}{2} \int_{a}^{+\infty} e^{c t} u^{\prime}(t)^{2} d t>0, B=\frac{c_{2}}{q+2} \int_{a}^{+\infty} e^{c t}|u(t)|^{q+2} d t>0$, since $u(t) \in H_{c, a}$ is nonzero function. We have that for every $\lambda \in[0,+\infty)$,

$$
\begin{aligned}
\mu_{[a,+\infty)}(u) & =\sup _{\lambda>0} J(\lambda u) \\
& \geq c_{4}(q) \frac{2^{\frac{2}{q}} \cdot q}{(q+2)^{1+\frac{2}{q}}}\left(\frac{\frac{1}{2} \int_{a}^{+\infty} e^{c t} u^{\prime}(t)^{2} d t}{\left(\frac{c_{2}}{q+2} \int_{a}^{+\infty} e^{c t}|u(t)|^{q+2} d t\right)^{\frac{2}{q+2}}}\right)^{\frac{q+2}{q}} \\
& \geq c_{5}(q)>0,
\end{aligned}
$$

where the constants $c_{4}(q)$ and $c_{5}(q)$ depend only on $q$. In the conclusion of the last inequality, we took into account (7). The lemma is proved.

Let $u(t) \in H_{c, a}$ be an arbitrary fixed nonzero function and the conditions of Lemma 2 be fulfilled. By (4) we have 


$$
\begin{aligned}
J(\lambda u) & =\frac{1}{2} \lambda^{2} \int_{a}^{+\infty} e^{c t} u^{\prime}(t)^{2} d t-\int_{a}^{+\infty} e^{c t} F(t, \lambda u(t)) d t \\
& \leq \lambda^{2} A_{1}-\left(\frac{c_{1}}{q+2} \int_{a}^{+\infty} e^{c t}|u(t)|^{q+2} d t\right) \lambda^{q+2} \\
& =A_{1} \lambda^{2}-B_{1} \lambda^{q+2} .
\end{aligned}
$$

Then $J(\lambda u)<0$ for sufficiently large $\lambda>0$. Since $J(0)=0$ and $J(\lambda u)$ is continuous function in $\lambda$, then $\mu(u)=\sup _{\lambda>0} J(\lambda u)$ is attained.

Moreover $\mu(u)=\mu(k u)$ for every constant $k>0$.

Thus for the given nonzero function $u(t) \in H_{c, a}$, there exists a positive number $\lambda_{0}>0$, such that

$$
\mu\left(\lambda_{0} u\right)=J\left(\lambda_{0} u\right)=\sup _{\lambda>0} J(\lambda u) .
$$

If we denote the function $\lambda_{0} u \in H_{c, a} \backslash\{0\}$ again by $u$, then the last equality can be written as

$$
\mu(u)=J(u) .
$$

We show that for any nonzero function $v \in H_{c, a}$, i.e. function belonging to $H_{c, a} \backslash\{0\}$, there exists a function $u \in H_{c, a} \backslash\{0\}$ such that $u=k v$, with suitable constant $k>0$, such that (8) holds. As in [9, p.2017] it follows that

$$
\left.\frac{\partial}{\partial \lambda} J(\lambda u)\right|_{\lambda=1}=\nabla J(u) u=\int_{a}^{+\infty} e^{c t}\left(u^{\prime}(t)^{2}-f(t, u(t)) u(t)\right) d t=0,
$$

which holds for critical points of $J(\lambda u)$ as a function of $\lambda$.

Lemma 3. Let the function $f(t, s) \in C^{1}\left(\mathbb{R}^{2}\right)$ satisfy the conditions (H1), (H2) and (H4) and $u \in H_{c, a}$ be nonzero function, for which (9) holds. Then

$$
J^{\prime \prime}(u)[u, u]<0 .
$$

Moreover there exists unique number $\lambda=\lambda(u)>0$ such that $\mu(u)=J(\lambda(u) u)$ and the function $u \rightarrow \lambda(u)$ is of class $C^{1}$.

Proof. We suppose that for the nonzero function $u \in H_{c, a}$, (9) holds, but $J^{\prime \prime}(u)[u, u] \geq 0$. Then

$$
\int_{a}^{+\infty} e^{c t}\left(u^{\prime}(t)^{2}-f_{s}(t, u(t)) u(t)^{2}\right) d t \geq 0
$$


Subtracting (10) from (9), we obtain that

$$
\int_{a}^{+\infty} e^{c t}\left(f_{s}(t, u(t)) u(t)^{2}-f(t, u(t)) u(t)\right) d t \leq 0 .
$$

Taking into account (H1) and (H2), we get

$$
\begin{aligned}
0 & \geq \int_{a}^{+\infty} e^{c t}\left(f_{s}(t, u(t)) u(t)^{2}-f(t, u(t)) u(t)\right) d t \\
& \geq \int_{a}^{+\infty} e^{c t}(A-1) f(t, u(t)) u(t) d t \\
& \geq c_{5}(A-1) \int_{a}^{+\infty} e^{c t}|u(t)|^{2+q} d t
\end{aligned}
$$

where the constant $c_{5}>0$ and $A>1$. Thus we proved that $\int_{a}^{+\infty} e^{c t}|u(t)|^{2+q} d t \leq$ 0 . But it is impossible for nonzero function $u(t)$. The obtained contradiction shows that the considered function $u(t)$ satisfies the inequality $J^{\prime \prime}(u)[u, u]<0$. The rest of the proof of the lemma is as in [9, Proposition 3.1]. Exactly, the unique number $\lambda=\lambda(u)>0$ such that $\mu(u)=J(\lambda(u) u)$, satisfies the equation

$$
\Phi(\lambda(u), u):=\nabla J(\lambda(u) u) \cdot u=0 .
$$

Also $\frac{\partial}{\partial \lambda} \Phi(\lambda, u)=\frac{\partial}{\partial \lambda}(\nabla J(\lambda u) . u)=J^{\prime \prime}(\lambda u)[u, u]<0$ for $\lambda=\lambda(u)$, where $\Phi$ is of class $C^{1}$ and the function $\lambda=\lambda(u)$ can be locally implicitly defined. From the implicit function theorem $\lambda(u)$ is of class $C^{1}$. Lemma 3 is proved.

The considerations in the proof of Lemma 3 show that

$$
\varphi^{+}(a,+\infty)=\inf _{N^{+}[a,+\infty)} J(u) .
$$

\section{Proof of the main result}

Let $\left\{u_{n}\right\} \subset N^{+}(a,+\infty)$ be a minimizing sequence for $\varphi^{+}(a,+\infty)$. Without loss of generality, we can suppose that

$$
\varphi^{+}(a,+\infty)+\varepsilon \geq J\left(u_{n}\right) \longrightarrow \varphi^{+}(a,+\infty), \quad n \rightarrow+\infty
$$

for sufficiently small number $\varepsilon>0$.

Proof of Theorem 1: 
Step 1. We have

$$
\begin{gathered}
c_{6} \int_{a}^{+\infty} e^{c t} u_{n}^{\prime}(t)^{2} d t \leq J\left(u_{n}\right) \leq c_{7} \int_{a}^{+\infty} e^{c t} u_{n}^{\prime}(t)^{2} d t, \\
c_{8} \int_{a}^{+\infty} e^{c t} F\left(t, u_{n}(t)\right) d t \leq J\left(u_{n}\right) \leq c_{9} \int_{a}^{+\infty} e^{c t} F\left(t, u_{n}(t)\right) d t
\end{gathered}
$$

for some positive constants $c_{i}, i=6,7,8,9$. Since $\nabla J\left(u_{n}\right) \cdot u_{n}=0$, as in (9),

$$
\int_{a}^{+\infty} e^{c t}\left(u_{n}^{\prime}(t)^{2}-f\left(t, u_{n}(t)\right) u_{n}(t)\right) d t=0 .
$$

We have $J\left(u_{n}\right)=\int_{a}^{+\infty} e^{c t}\left(\frac{1}{2} u_{n}^{\prime}(t)^{2}-F\left(t, u_{n}(t)\right)\right) d t$. By (H3) and (4),

$$
\begin{aligned}
2 J\left(u_{n}\right) & =\int_{a}^{+\infty} e^{c t}\left(f\left(t, u_{n}(t)\right) u_{n}(t)-2 F\left(t, u_{n}(t)\right)\right) d t \\
& \geq \alpha \int_{a}^{+\infty} e^{c t} F\left(t, u_{n}(t)\right) d t \geq \frac{\alpha c_{1}}{q+2} \int_{a}^{+\infty} e^{c t}\left|u_{n}(t)\right|^{2+q} d t .
\end{aligned}
$$

This inequality shows that $J\left(u_{n}\right) \geq 0$. Hence

$$
\begin{aligned}
\frac{1}{2} \int_{a}^{+\infty} e^{c t} u_{n}^{\prime}(t)^{2} d t & =\int_{a}^{+\infty} e^{c t} F\left(t, u_{n}(t)\right) d t+J\left(u_{n}\right) \\
& \leq \frac{c_{2}}{q+2} \int_{a}^{+\infty} e^{c t}\left|u_{n}(t)\right|^{2+q} d t+J\left(u_{n}\right) \\
& \leq\left(1+\frac{2 c_{2}}{\alpha c_{1}}\right) J\left(u_{n}\right)
\end{aligned}
$$

and

$$
J\left(u_{n}\right) \geq \frac{\alpha c_{1}}{2 \alpha c_{1}+4 c_{2}} \int_{a}^{+\infty} e^{c t} u_{n}^{\prime}(t)^{2} d t .
$$

By the definition of $J\left(u_{n}\right)$ and $(4), J\left(u_{n}\right) \leq \frac{1}{2} \int_{a}^{+\infty} e^{c t} u_{n}^{\prime}(t)^{2} d t$. Thus, we proved the first inequality (12) with $c_{6}=\frac{\alpha c_{1}}{2 \alpha c_{1}+4 c_{2}}$ and $c_{7}=\frac{1}{2}$. The inequality (13) holds with $c_{8}=\frac{\alpha}{2}$ and $c_{9}=\frac{c_{2}(q+2)}{2 c_{1}}$ by (H1) and (4) since

$$
\begin{aligned}
2 J\left(u_{n}\right) & \leq \int_{a}^{+\infty} e^{c t} u_{n}^{\prime}(t)^{2} d t=\int_{a}^{+\infty} e^{c t} f\left(t, u_{n}(t)\right) u_{n}(t) d t \\
& \leq \frac{c_{2}(q+2)}{c_{1}} \int_{a}^{+\infty} e^{c t} F\left(t, u_{n}(t)\right) d t
\end{aligned}
$$


These assertions show that $\int_{a}^{+\infty} e^{c t} u_{n}^{\prime}(t)^{2} d t$ and $\int_{a}^{+\infty} e^{c t}\left|u_{n}(t)\right|^{2+q} d t$ are bounded by constant, which does not depend on $n$. From the inequality (6), the same is true for $\int_{a}^{+\infty} e^{c t} u_{n}(t)^{2} d t$. Hence

$$
\int_{a}^{+\infty} e^{c t}\left(u_{n}(t)^{2}+u_{n}^{\prime}(t)^{2}\right) d t
$$

and

$$
\int_{a}^{+\infty} e^{c t}\left|u_{n}(t)\right|^{2+q} d t
$$

are bounded by a constant, which does not depend on $n$. Then, the sequence $\left\{u_{n}\right\}$ is bounded in $H_{c, a}$ equipped by the norm $\|u\|_{H_{c, a}}=\left(\int_{a}^{+\infty} e^{c t} u^{\prime}(t)^{2} d t\right)^{\frac{1}{2}}$.

Step 2. There exists a function $u_{0} \in H_{c, a}^{0}:=H_{c, a} \cap H_{0}^{1}[a,+\infty)$ and subsequence $\left\{u_{n_{k}}\right\}$ still denoted by $\left\{u_{n}\right\}$, such that $u_{n} \longrightarrow u_{0}$ for $n \rightarrow+\infty$ in the week $H_{c, a}^{0}-$ topology; $u_{n} \longrightarrow u_{0}$ for $n \rightarrow+\infty$ in the strong $L^{2}-$ topology and on any bounded and closed subinterval of $[a,+\infty)$. Moreover $u_{0} \geq 0$ is nonzero function. Let us remind that $\left\{u_{n}\right\} \subset N^{+}(a,+\infty)$ and then $u_{n} \geq 0, n=1,2, \ldots$. Hence $u_{0} \geq 0$.

We will prove that $u_{0}$ is nonzero function. Suppose the contrary, i.e., that $u_{0} \equiv 0$. This means that $u_{n} \longrightarrow 0$ for $n \rightarrow+\infty$ in the week $H_{c, a}^{0}-$ topology and $u_{n} \longrightarrow 0$ for $n \rightarrow+\infty$ in the strong $L^{2}$ - topology on any bounded and closed subinterval of $[a,+\infty)$. Let $b>a$ be an arbitrary number. From [1, p.321],

$$
\left|u_{n}(b)\right| \leq\left(\frac{e^{-c b}}{c} \int_{b}^{+\infty} e^{c t} u_{n}^{\prime}(t)^{2} d t\right)^{\frac{1}{2}} \leq c_{10} e^{-\frac{c}{2} b}, \quad \forall b>a
$$

and the constant $c_{10}>0$ does not depend on $n \in \mathbb{N}$ and $b$. Let $b \in(a,+\infty)$ be a fixed (sufficiently large) number. Then for every $\varepsilon>0$, there exists $n_{0} \in \mathbb{N}$, depending on $b$ and $\varepsilon$, such that

$$
\int_{a}^{b} e^{c t} u_{n}(t)^{2} d t \leq e^{c b} \int_{a}^{b} u_{n}(t)^{2} d t<\varepsilon, \quad \forall n \geq n_{0} .
$$

From (14), replacing $b$ by $t,\left|u_{n}(t)\right| \leq c_{10} e^{-\frac{c}{2} t}, \forall t \in[a,+\infty)$ and for some $n_{1}>n_{0}, n_{1} \in \mathbb{N}$, depending on $b$, we get by $q>0$

$$
\int_{a}^{b} e^{c t}\left|u_{n}(t)\right|^{2+q} d t \leq c_{10} \int_{a}^{b} e^{c t}\left|u_{n}(t)\right|^{2} d t<\frac{\varepsilon}{2}, \forall n \geq n_{1} .
$$

From (14), we have

$$
\int_{b}^{+\infty} e^{c t}\left|u_{n}(t)\right|^{2+q} d t \leq \max _{t \in[b,+\infty)}\left|u_{n}(t)\right|^{q} \cdot \int_{a}^{+\infty} e^{c t}\left|u_{n}(t)\right|^{2} d t \leq c_{11} e^{-\frac{c}{2} b q}
$$


and the constant $c_{11}>0$ does not depend on $b \in(a,+\infty)$ and $n \in \mathbb{N}$. Now let $\varepsilon>0$ be a fixed, sufficiently small number. We choose the number $b \in(a,+\infty)$ so large, such that $c_{11} e^{-\frac{c}{2} b q}<\frac{\varepsilon}{2}$. Then by (16),

$$
\int_{b}^{+\infty} e^{c t}\left|u_{n}(t)\right|^{2+q} d t<\frac{\varepsilon}{2}, \quad \forall n \in \mathbb{N} .
$$

We choose the number $n_{1} \in \mathbb{N}, n_{1}>n_{0}$, such that (15) holds and hence $\int_{a}^{+\infty} e^{c t}\left|u_{n}(t)\right|^{2+q} d t<\varepsilon, \forall n \geq n_{1}$. Thus we prove that

$$
\lim _{n \rightarrow+\infty} \int_{a}^{+\infty} e^{c t}\left|u_{n}(t)\right|^{2+q} d t=0 .
$$

By Step 1, (4) and (13) it follows $\lim _{n \rightarrow+\infty} J\left(u_{n}\right)=0$. This contradicts to Lemma 2 , according which $J\left(u_{n}\right)=\mu_{[a,+\infty)}\left(u_{n}\right) \geq C>0$. The contradiction shows that $u_{0}$ is a nonzero function.

Step 3. $\int_{a}^{+\infty} e^{c t} u_{n}^{\prime}(t)^{2} d t \geq \int_{a}^{+\infty} e^{c t} u_{0}^{\prime}(t)^{2} d t+o(1)$.

We have

$$
\begin{gathered}
\int_{a}^{+\infty} e^{c t} u_{n}^{\prime}(t)^{2} d t=\int_{a}^{+\infty} e^{c t}\left[u_{0}^{\prime}(t)+\left(u_{n}^{\prime}(t)-u_{0}^{\prime}(t)\right)\right]^{2} d t \\
=\int_{a}^{+\infty} e^{c t} u_{0}^{\prime}(t)^{2} d t+2 \int_{a}^{+\infty} e^{c t}\left(u_{n}^{\prime}(t)-u_{0}^{\prime}(t)\right) u_{0}^{\prime}(t) d t \\
\quad+\int_{a}^{+\infty} e^{c t}\left(u_{n}^{\prime}(t)-u_{0}^{\prime}(t)\right)^{2} d t .
\end{gathered}
$$

By $u_{n} \longrightarrow u_{0}$ for $n \rightarrow+\infty$ weakly in $H_{c, a}^{0}$ we have

$$
\begin{aligned}
& \lim _{n \rightarrow+\infty} \int_{a}^{+\infty} e^{c t}\left(u_{n}^{\prime}(t)-u_{0}^{\prime}(t)\right) u_{0}^{\prime}(t) d t \\
= & \lim _{n \rightarrow+\infty}\left\langle u_{n}^{\prime}(t)-u_{0}^{\prime}(t), u_{0}^{\prime}(t)\right\rangle_{H_{c, a}}=0 .
\end{aligned}
$$

Then

$$
2 \int_{a}^{+\infty} e^{c t}\left(u_{n}^{\prime}(t)-u_{0}^{\prime}(t)\right) u_{0}^{\prime}(t) d t=o(1)
$$

and by (17),

$$
\int_{a}^{+\infty} e^{c t} u_{n}^{\prime}(t)^{2} d t \geq \int_{a}^{+\infty} e^{c t} u_{0}^{\prime}(t)^{2} d t+o(1)
$$

Step 4. $\lim _{n \rightarrow+\infty} \int_{a}^{+\infty} e^{c t} F\left(t, u_{n}(t)\right) d t=\int_{a}^{+\infty} e^{c t} F\left(t, u_{0}(t)\right) d t$.

As in Step 2, replacing $u_{n}$ by $u_{n}-u_{0}$, we can prove that

$$
\lim _{n \rightarrow+\infty} \int_{a}^{+\infty} e^{c t}\left|u_{n}(t)-u_{0}(t)\right|^{2+q} d t=0 .
$$


By $(\mathrm{H} 1)$ and $u_{n}(t) \geq 0, u_{0}(t) \geq 0$,

$$
\begin{aligned}
\left|F\left(t, u_{n}(t)\right)-F\left(t, u_{0}(t)\right)\right| & =\left|\int_{u_{0}(t)}^{u_{n}(t)} f(t, \tau) d \tau\right| \leq c_{2}\left|\int_{u_{0}(t)}^{u_{n}(t)} \tau^{1+q} d \tau\right| \\
& =\frac{c_{2}}{q+2}\left|u_{n}(t)^{2+q}-u_{0}(t)^{2+q}\right|
\end{aligned}
$$

Hence

$$
\begin{aligned}
& \left|\int_{a}^{+\infty} e^{c t} F\left(t, u_{n}(t)\right) d t-\int_{a}^{+\infty} e^{c t} F\left(t, u_{0}(t)\right) d t\right| \\
\leq & \frac{c_{2}}{q+2} \int_{a}^{+\infty} e^{c t}\left|u_{n}(t)^{2+q}-u_{0}(t)^{2+q}\right| d t \\
\leq & K_{1} \int_{a}^{+\infty} e^{c t}\left|u_{n}(t)-u_{0}(t)\right|\left(u_{n}(t)^{1+q}+u_{0}(t)^{1+q}\right) d t .
\end{aligned}
$$

Using the Hölder inequality, it is easy to obtain that

$$
\begin{aligned}
& \left|\int_{a}^{+\infty} e^{c t} F\left(t, u_{n}(t)\right) d t-\int_{a}^{+\infty} e^{c t} F\left(t, u_{0}(t)\right) d t\right| \\
\leq & K_{1}\left(\int_{a}^{+\infty} e^{c t}\left(u_{n}(t)^{1+q}+u_{0}(t)^{1+q}\right)^{\frac{2+q}{1+q}} d t\right)^{\frac{1+q}{2+q}} \\
& \left(\int_{a}^{+\infty} e^{c t}\left|u_{n}(t)-u_{0}(t)\right|^{2+q} d t\right)^{\frac{1}{2+q}} \\
\leq & K_{2}\left(\int_{a}^{+\infty} e^{c t} u_{n}(t)^{2+q} d t+\int_{a}^{+\infty} e^{c t} u_{0}(t)^{2+q} d t\right)^{\frac{1+q}{2+q}} \\
& \left(\int_{a}^{+\infty} e^{c t}\left|u_{n}(t)-u_{0}(t)\right|^{2+q} d t\right)^{\frac{1}{2+q}} \\
\leq & K_{3}\left(\int_{a}^{+\infty} e^{c t}\left|u_{n}(t)-u_{0}(t)\right|^{2+q} d t\right)^{\frac{1}{2+q}},
\end{aligned}
$$

where $K_{j}, j=1,2,3$ are constants not depending on $u$ and $n$. Taking into account (18), we obtain the assertion of Step 4.

Step 5. There exists a nonzero function $u_{+} \in N^{+}(a,+\infty)$, which $u_{+} \geq 0$ and $J_{[a,+\infty)}\left(u_{+}\right)=\varphi^{+}(a,+\infty)=\inf _{N^{+}(a,+\infty)} J_{[a,+\infty)}(u)>0$. 
From (4), Step 3 and Step 4 it follows that $J\left(u_{n}\right) \geq J\left(u_{0}\right)+o(1)$ (i.e., the functional $J$ is weakly lower semi continuous). Hence the inequality $J_{[a,+\infty)}\left(\lambda u_{0}\right) \leq$ $J_{[a,+\infty)}\left(\lambda u_{n}\right)+o(1), \forall \lambda>0$ holds.

Define

$$
u_{+}:=\lambda\left(u_{0}\right) u_{0}
$$

We obtain,that $u_{+}$satisfies the conditions of Step 5 .

We will prove that $u_{+}>0$ on $(a,+\infty)$. For this purpose, we adapt the proof of Theorem 3.1 of [9, pp. 2019-2020] to our case.

Claim 1. $u_{+} \in C^{1}(a,+\infty)$. We assume that there exists $\tau \in(a,+\infty)$, for which $u_{+}(\tau)=0$ and $u_{+}^{\prime}(t)$ is not continuous for $t=\tau$. Let $u_{+}^{\prime}(\tau-0)<0$ and the constants $\rho>0$ and $\varepsilon>0$ are sufficiently small numbers. For $\lambda \in$ $[1-\varepsilon, 1+\varepsilon]$ we consider the class of problems

$$
\inf \left\{\begin{array}{cc}
v(\tau-\rho)=\lambda u_{+}(\tau-\rho) \\
J_{[\tau-\rho, \tau+\rho]}(v): v \in H^{1}(\tau-\rho, \tau+\rho), & v(\tau+\rho)=\lambda u_{+}(\tau+\rho) \\
\|v\|_{\infty} \leq 1
\end{array}\right\}
$$

where $\tau-\rho, \tau+\rho \in(a,+\infty)$. We have

$$
\begin{aligned}
\frac{d^{2}}{d \lambda^{2}} J_{[\tau-\rho, \tau+\rho]}( & u+\lambda \varphi)_{\lambda=0}=\int_{\tau-\rho}^{\tau+\rho} e^{c t}\left(\varphi^{\prime}(t)^{2}-f_{s}^{\prime}(t, u(t)) \varphi(t)^{2}\right) d t \\
& \geq \int_{\tau-\rho}^{\tau+\rho} e^{c t} \varphi^{\prime}(t)^{2} d t-c_{13} \int_{\tau-\rho}^{\tau+\rho} e^{c t} \varphi(t)^{2} d t \\
& \geq\left(\frac{c_{14}}{\rho^{2}}-c_{3}\right) \int_{\tau-\rho}^{\tau+\rho} e^{c t} \varphi(t)^{2} d t>0,
\end{aligned}
$$

where the constants $c_{13}:=\sup \left\{f_{s}^{\prime}(t, u): a \leq t<+\infty,-1 \leq u \leq 1\right\}, c_{14}>0$, and the function $\varphi(t)$ vanishes on at least one point $t=t_{0} \in[\tau-\rho, \tau+\rho]$. As in [9, p. 2026, Lemma 5.1], we can conclude, that $J$ is strictly convex, and thus the minimum of (19) is uniquely achieved by a function $v_{\lambda}$. We will show that $v_{\lambda}$ satisfies Eq. (1.) and $\lim _{\rho \rightarrow 0}\left\|v_{\lambda}\right\|_{H^{1}(\tau-\rho, \tau+\rho)}=0$. For this purpose, we need to prove that $\left|v_{\lambda}(t)\right|<1$ and $v_{\lambda}(t) \geq 0$ for every $t \in[\tau-\rho, \tau+\rho]$.

First, we prove that $\left\|v_{\lambda}\right\|_{\infty}=\max _{t \in[\tau-\rho, \tau+\rho]}\left|v_{\lambda}(t)\right|<1$. Suppose the contrary, $\left\|v_{\lambda}\right\|_{\infty}=1$. By inclusion $H^{1}(\tau-\rho, \tau+\rho) \subset C[\tau-\rho, \tau+\rho], u_{+} \in$ $C[\tau-\rho, \tau+\rho]$ and $v_{\lambda} \in C[\tau-\rho, \tau+\rho]$. By $u_{+}(\tau)=0, v_{\lambda}(\tau \pm \rho)=$ $\lambda u_{+}(\tau \pm \rho)=o(1)$ for $\rho \rightarrow 0+$. If $v_{\lambda}\left(\tau_{1}\right)= \pm 1$ for some $\tau_{1} \in(\tau-\rho, \tau+\rho)$, then 


$$
\begin{aligned}
& \quad\left|v_{\lambda}\left(\tau_{1}\right)-v_{\lambda}(\tau-\rho)\right|=1-o(1)=\left|\int_{\tau-\rho}^{\tau_{1}} v_{\lambda}^{\prime}(t) d t\right| \\
& \leq \int_{\tau-\rho}^{\tau+\rho} e^{-\frac{c t}{2}} e^{\frac{c t}{2}}\left|v_{\lambda}^{\prime}(t)\right| d t \\
& \leq\left(\int_{\tau-\rho}^{\tau+\rho} e^{-c t} d t\right)^{\frac{1}{2}}\left(\int_{\tau-\rho}^{\tau+\rho} e^{c t} v_{\lambda}^{\prime}(t)^{2} d t\right)^{\frac{1}{2}} \\
& =\left(\frac{e^{-c(\tau-\rho)}-e^{-c(\tau+\rho)}}{c}\right)^{\frac{1}{2}}\left(\int_{\tau-\rho}^{\tau+\rho} e^{c t} v_{\lambda}^{\prime}(t)^{2} d t\right)^{\frac{1}{2}} \\
& \quad \leq c_{15 \sqrt{\rho}}\left(\int_{\tau-\rho}^{\tau+\rho} e^{c t} v_{\lambda}^{\prime}(t)^{2} d t\right)^{\frac{1}{2}},
\end{aligned}
$$

where the constant $c_{15}>0$ is close to $\left(2 e^{-c \tau}\right)^{\frac{1}{2}}$ for small $\rho$, i.e. $c_{15}$ depends only on $\tau$. Since $1-o(1) \geq \frac{1}{\sqrt{2}}$ for small $\rho$, then

$$
\int_{\tau-\rho}^{\tau+\rho} e^{c t} v_{\lambda}^{\prime}(t)^{2} d t \geq \frac{1}{2 c_{15}^{2} \rho} .
$$

Since $F\left(t, v_{\lambda}\right)$ is bounded for $t \in[\tau-\rho, \tau+\rho]$, and $\frac{1}{2 c_{15}^{2} \rho}-C \geq \frac{c_{16}}{\rho}$ for small $\rho>0$, it implies

$$
J_{[\tau-\rho, \tau+\rho]}\left(v_{\lambda}\right) \geq \frac{c_{16}}{\rho} .
$$

Remind that $u_{+} \in H^{1}(\tau-\rho, \tau+\rho)$ and $u_{+}(\tau)=0$. As above

$$
\begin{aligned}
\max _{[\tau-\rho, \tau+\rho]}\left|u_{+}(t)\right| & =\max _{[\tau-\rho, \tau+\rho]}\left|u_{+}(t)-u_{+}(\tau)\right| \\
& \leq c_{15} \sqrt{\rho}\left(\int_{\tau-\rho}^{\tau+\rho} e^{c t} u_{+}^{\prime}(t)^{2} d t\right)^{\frac{1}{2}}=o(1) \sqrt{\rho},
\end{aligned}
$$

because $u_{+} \in H_{c, a}^{0}$ and then $\left(\int_{\tau-\rho}^{\tau+\rho} e^{c t} u_{+}^{\prime}(t)^{2} d t\right)^{\frac{1}{2}}=o(1)$ when $\rho>0$ is sufficiently small. Thus

$$
\left|\lambda u_{+}(\tau \pm \rho)\right|=o(\sqrt{\rho}), \forall \lambda \in[1-\varepsilon, 1+\varepsilon] .
$$

Now we consider the linear function

$$
w(t)=u_{+}(\tau-\rho)-\frac{u_{+}(\tau-\rho)-u_{+}(\tau+\rho)}{2 \rho}(t-\tau+\rho) .
$$


Evidently

$$
w(t-\rho)=u_{+}(\tau-\rho),
$$

$w(t+\rho)=u_{+}(\tau+\rho)$ and $w^{\prime}(t)=\frac{u_{+}(\tau+\rho)-u_{+}(\tau-\rho)}{2 \rho}$. As above we have

$$
\begin{aligned}
\left|u_{+}(\tau-\rho)-u_{+}(\tau+\rho)\right| & =\left|\int_{\tau-\rho}^{\tau+\rho} u^{\prime}(s) d s\right| \\
& \leq c_{15} \sqrt{\rho}\left(\int_{\tau-\rho}^{\tau+\rho} e^{c t} u_{+}^{\prime}(t)^{2} d t\right)^{\frac{1}{2}}=\sqrt{\rho} . o(1),
\end{aligned}
$$

because $u_{+} \in H_{c, a}^{0}$. Then $\left(\int_{\tau-\rho}^{\tau+\rho} e^{c t} u_{+}^{\prime}(t)^{2} d t\right)^{\frac{1}{2}}=o(1)$ when $\rho>0$ is sufficiently small. Thus

$$
\begin{aligned}
\left|w^{\prime}(t)\right| & \leq \frac{C}{\sqrt{\rho}} \cdot o(1) \\
0 & \leq \int_{\tau-\rho}^{\tau+\rho} e^{c t} w^{\prime}(t)^{2} d t \leq \frac{C}{\rho} \cdot o(1) \int_{\tau-\rho}^{\tau+\rho} e^{c t} d t \leq o(1) .
\end{aligned}
$$

Also,

$$
\begin{aligned}
|w(t)| & \leq \max \left(u_{+}(\tau-\rho), u_{+}(\tau+\rho)\right) \\
& \leq \max _{[\tau-\rho, \tau+\rho]}\left|u_{+}(t)\right|=o(1) \sqrt{\rho}, \quad \forall t \in[\tau-\rho, \tau+\rho] .
\end{aligned}
$$

Hence $\|w\|_{H^{1}[\tau-\rho, \tau+\rho]}=o(1)$ and

$$
J_{[\tau-\rho, \tau+\rho]}(w)=o(1), J_{[\tau-\rho, \tau+\rho]}(\lambda w)=o(1) \forall \lambda \in[1-\varepsilon, 1+\varepsilon] .
$$

From $(21)$ and $(22), J_{[\tau-\rho, \tau+\rho]}(\lambda w)<<J_{[\tau-\rho, \tau+\rho]}\left(v_{\lambda}\right), \forall \lambda \in[1-\varepsilon, 1+\varepsilon]$ for sufficiently small $\rho>0$, (where $a<<b$ means that $\frac{a}{b}=o(1)$ ). The last inequality contradicts to the fact, that infimum in (19) is attained by the function $v_{\lambda}$. The contradiction is due to the assumption, that $\left\|v_{\lambda}\right\|_{\infty}=1$. Therefore $\left\|v_{\lambda}\right\|_{\infty}<1$ and thus $v_{\lambda}$ is a solution of Eq.(1).

Now we will prove that $v_{\lambda} \geq 0$ for $t \in[\tau-\rho, \tau+\rho]$. Suppose the contrary. Then changing the sign, $v_{\lambda}$ vanish at some point of $[\tau-\rho, \tau+\rho]$ and by $v_{\lambda}(\tau \pm \rho)=\lambda u_{+}(\tau \pm \rho) \geq 0$, it follows that $v_{\lambda}^{\prime}$ also vanish at some other point of $[\tau-\rho, \tau+\rho]$. Since $v_{\lambda}$ satisfies $(1)$, then

$$
\left(e^{c t} v_{\lambda}^{\prime}\right)^{\prime}+e^{c t} f\left(t, v_{\lambda}\right)=0 \Longrightarrow v_{\lambda}^{\prime}(t)=-\int_{t_{1}}^{t} e^{-c(t-s)} f\left(s, v_{\lambda}(s)\right) d s
$$


where $t_{1} \in[\tau-\rho, \tau+\rho]$ is such that $v_{\lambda}^{\prime}\left(t_{1}\right)=0$. Then

$$
\begin{aligned}
\left|v_{\lambda}^{\prime}(t)\right| & \leq \int_{t_{1}}^{t} e^{-c(t-s)}\left|f\left(s, v_{\lambda}(s)\right)\right| d s \\
& \leq c_{17} \int_{\tau-\rho}^{\tau+\rho} e^{-c(t-s)} d s=c_{17} \frac{e^{-c(\tau-\rho)}-e^{-c(\tau+\rho)}}{c} \leq c_{18} \rho,
\end{aligned}
$$

because $f\left(s, v_{\lambda}(s)\right)$ is bounded, for $s \in[\tau-\rho, \tau+\rho]$. Thus

$$
\left|v_{\lambda}^{\prime}(t)\right| \leq c_{18} \rho \forall t \in[\tau-\rho, \tau+\rho]
$$

where the constant $c_{18}>0$ depends only on $\tau$. Recall that $u_{+}(\tau)=0$ and $u_{+}^{\prime}(\tau-0)<0$. Then for $\rho>0$ sufficiently small, $u_{+}(\tau-\rho)=u_{+}(\tau)-$ $\rho u_{+}^{\prime}(\tau-0)=-\rho u_{+}^{\prime}(\tau-0)$. Hence

$$
v_{\lambda}(\tau-\rho)=\lambda u_{+}(\tau-\rho)=-\lambda \rho u_{+}^{\prime}(\tau-0),
$$

i.e. $c_{19} \rho \leq v_{\lambda}(\tau-\rho) \leq c_{20} \rho$ for $\rho>0$ sufficiently small. Then $v_{\lambda}(t)=$ $v_{\lambda}(\tau-\rho)+\int_{\tau-\rho}^{t} v_{\lambda}^{\prime}(s) d s$ and from $(23)$

$$
\left|\int_{\tau-\rho}^{t} v_{\lambda}^{\prime}(s) d s\right| \leq c_{18} \rho^{2}<<v_{\lambda}(\tau-\rho), \quad \forall t \in[\tau-\rho, \tau+\rho] .
$$

Thus $v_{\lambda}(t)$ cannot change the sign when $t \in[\tau-\rho, \tau+\rho]$. This consideration is true also, when $u_{+}^{\prime}(\tau-0)=-\infty$, because $v_{\lambda}(\tau-\rho) \geq C \rho$ for sufficiently large constant $C>0$ and $v_{\lambda}(t)$ does not change the sign again. We prove, that $v_{\lambda} \geq 0$ for $t \in[\tau-\rho, \tau+\rho]$.

Note that $v_{\lambda}(t) \in C^{1}(\tau-\rho, \tau+\rho)$, because $v_{\lambda}(t)$ satisfies Eq. (1) for $t \in(\tau-\rho, \tau+\rho)$. Now we define the function

$$
\tilde{u}_{\lambda}(t):=\left\{\begin{array}{cc}
v_{\lambda}(t), & t \in[\tau-\rho, \tau+\rho], \\
\lambda u_{+}(t), & t \in[a,+\infty) \backslash[\tau-\rho, \tau+\rho] .
\end{array}\right.
$$

We should note that $\tilde{u}_{\lambda}(t) \geq 0 \quad \forall t \in[a,+\infty)$ and $\tilde{u}_{\lambda}(t) \in H_{c, a}^{0}$, because $v_{\lambda}(t)$ satisfies the boundary conditions in (19). We will prove, that $\tilde{u}_{\lambda} \rightarrow \lambda u_{+}$in $H_{c, a}^{0}$, when $\rho \rightarrow 0$. Indeed, from $(22), J_{[\tau-\rho, \tau+\rho]}\left(v_{\lambda}\right) \leq J_{[\tau-\rho, \tau+\rho]}(w) \leq o(1)$ and since

$$
\left\|v_{\lambda}\right\|_{\infty}<1 \Longrightarrow \int_{\tau-\rho}^{\tau+\rho} F\left(t, v_{\lambda}(t)\right) d t<\frac{2 c_{2}}{q+2} \rho
$$

then $\int_{\tau-\rho}^{\tau+\rho} e^{c t} v_{\lambda}^{\prime}(t)^{2} d t=o(1)$ when $\rho \rightarrow 0$. This proves that $\tilde{u}_{\lambda} \rightarrow \lambda u_{+}$in $H_{c, a}^{0}$, when $\rho \rightarrow 0$. Further the proof that $u_{+} \in C^{1}(a,+\infty)$ holds as in [9, p.2020]. Finally we should note that there exists finite right derivative $u_{+}^{\prime}(a+0)$. Indeed, 
if $u_{+}(t) \equiv 0$ in a small right neighborhood of $a$, then obviously $u_{+}^{\prime}(a+0)=0$. If $u_{+}(t)>0$ in a small right neighborhood of $a$, then $u_{+}(t)$ satisfies (1) and it is easy to show that

$$
\begin{aligned}
u_{+}^{\prime}(a+0) & :=\lim _{t \rightarrow a+0} u_{+}^{\prime}(t) \\
= & e^{-c\left(a-t_{0}\right)}\left[u_{+}^{\prime}\left(t_{0}\right)+\int_{a}^{t_{0}} e^{c\left(s-t_{0}\right)} f\left(s, u_{+}(s)\right) d s\right],
\end{aligned}
$$

where $t_{0}>a$ is a point, close enough to $a$. Thus we prove, that $u_{+} \in$ $C^{1}[a,+\infty)$.

Claim 2. $u_{+}(t)>0, \forall t \in[a,+\infty)$.

We assume that $u_{+}\left(t_{0}\right)=0$ for some $t_{0}>a$, and $u_{+}(t)>0$ in a small right (left) neibourghood of $t_{0}$. Since $u_{+} \geq 0$, then $t_{0}$ is a point of local minimum for $u_{+}$and since $u_{+} \in C^{1}(a,+\infty)$, then $u_{+}^{\prime}\left(t_{0}\right)=0$. Since $f(t, 0) \equiv 0$, then $u \equiv 0$ is a solution of (1) for the Cauchy conditions $u\left(t_{0}\right)=u^{\prime}\left(t_{0}\right)=0$ and from the uniqueness theorem of the local Cauchy problem, follows that $u_{+}(t) \equiv 0$ in a small neibourhood of $t_{0}$. Thus it implies $u_{+}(t)=0, \forall t \in[a,+\infty)$, which contradicts to the definition of $u_{+}$as a nonzero function. This contradiction proves the assertion of Claim 2. Theorem 1 is proved.

\section{Acknowledgements}

The second author is supported by the Bulgarian National Science Fund under Project DN 12/4 'Advanced analytical and numerical methods for nonlinear differential equations with applications in finance and environmental pollution', (2017). His work is also in frames of the working program under the bilateral agreement between Bulgarian Academy of Sciences and Serbian Academy of Sciences and Arts (2020-2022).

\section{References}

[1] M. Arias, J. Campos, A.M. Robles-Perez, L. Sanchez, Fast and heteroclinic solutions for a second order ODE related to Fisher-Kolmogorov's equation. Calculus of Variations, 21 (2004), 319-334.

[2] D.G. Aronson, H.F. Weinberger, Nonlinear diffusion in population genetics, combustion and nerve pulse propagation, In: Partial Differential Equations and Related Topics, Lecture Notes in Math., 446, Springer, New York (1975), 5-49. 
[3] A.N. Kolmogorov, I.G. Petrovsky, N.S. Piskunov. Étude de l'équation de la diffusion avec croissancede la quantite de matiere et son application a une problème biologique, Bull. Univ. d'Etat a Moscou, Ser. Internationale A, 1 (1937), 1-26.

[4] Y. Li, Z.Q. Wang, Gluing approximate solutions of minimum type on the Nehari manifold, In: Proc. USA-Chile Workshop on Nonlinear Analysis (Via del Mar-Valparaiso, 2000), Electron. J. Diff. Eqns Conf., 6 (2000), 215-223.

[5] C. Marcelli, F. Papalini, Heteroclinic connections for fully nonlinear nonautonomous second order differential equations, J. Differential Equations, 241 (2007), 160-183.

[6] J. Mawhin, Critical Point Theory and Applications to Nonlinear Differential Equation, Lectures given at the VIGRE Minicourse on Varitional Method and Nonlinear PDE, University of Utah, May 28-June 8, 2002.

[7] Z. Nehari, Characteristic values associated with a class of nonlinear second order differential equations, Acta Math., 105 (1961), 141-175.

[8] A. Szulkin, The metod of Nehari manifold revisited (Progress in variational problems: New trends of geometric gradient flow and critical point theory), Kyoto University Research Information Repository (2011), 89-102.

[9] G. Verzini, Bounded solutions to superlinear ODEs: a variational approach, Nonlinearity, 16 (2003), 2013-2028.

[10] V.A. Volpert, Y.M. Suhov, Stationary solutions of non-autonomous Kolmogorov-Petrovsky-Piskunov equations, Ergodic Theory Dynam. System, 19 (1999). 
